The Journal of Physical Chemistry C

January 2017, Volume 121, Issue 4, Pages 2402-2409

http://dx.doi.org/10.1021/acs.jpcc.6b11779

http://archimer.ifremer.fr/doc/00367/47865/

(c) 2017 American Chemical Society

\title{
Plasmonic Properties of Aluminium Nanocylinders in the Visible Range
}

\author{
Gillibert Raymond ${ }^{1,2}$, Colas Florent ${ }^{3}$, Yasukuni Ryohei ${ }^{1}$, Picardi Gennaro ${ }^{1}$, \\ Lamy de la Chapelle Marc ${ }^{1,}$
}

${ }^{1}$ Universite Paris 13, Sorbonne Paris Cite, Laboratoire CSPBAT, CNRS, (UMR 7244), 74 rue Marcel Cachin, F-93017 Bobigny, France,

${ }^{2}$ HORIBA Jobin Yvon S.A.S. 231 rue de Lille 59650 Villeneuve d'Ascq, France

${ }^{3}$ Laboratoire Ditection, Capteurs et Mesures, Centre Bretagne - ZI de la Pointe du Diable - CS 10070 29280 Plouzané, France

* Corresponding author : Marc Lamy de la Chapelle, email address :

marc.lamydelachapelle@univ-paris13.fr

\begin{abstract}
:
Plasmonic and surface enhanced Raman scattering (SERS) studies have been performed on aluminium nanocylinders arrays of different diameters. We observed sharps localised surface plasmon resonance (LSPR) peaks that can be tuned on the whole visible range and having the same behaviour than gold nanocylinders. The near-field enhancement was measured by SERS on probe molecules as well as on the indium tin oxide (ITO) substrate using two excitation wavelengths: 660 and $785 \mathrm{~nm}$. No SERS signal of the probe molecul
\end{abstract}


Aluminium as plasmonic material has attracted considerable attention in the recent years and several plasmonic studies have been performed on aluminium nanostructures. Indeed, the real part of the $\mathrm{Al}$ dielectric constant is strongly negative and allows having a plasmon resonance from the ultra-violet to the far infra-red. As a consequence, the aluminium appears to be usable in plasmonics on a very wide spectral range. Moreover, its price is very low compared to gold or silver usually used in plasmonic devices which make $\mathrm{Al}$ a good candidate to reduce the cost of such systems for mass production. Plasmonic studies were conducted by C. Langhammer et al. ${ }^{1}$ on aluminium nanocylinders randomly distributed on a glass slide. Those substrates showed a tuneable resonance position depending on the disk diameter. It was also shown that a thin alumina oxide layer of a few manometers is quickly formed at the surface of the structures, its thickness slowly growing with time. The potential applications of such nanostructures to surface enhanced spectroscopy have also been studied. For instances, 
in 2007, C. Zhang et al. ${ }^{2}$ designed an aluminium substrate for surface enhanced Raman scattering (SERS) produced by anodisation and the SERS signal of protein and bacteria were recorded with a $514 \mathrm{~nm}$ laser line. Later, a strong SERS signal of crystal violet has been reported on a simple aluminium sputtered surface with a UV excitation (244 nm) by T. Dörfer et al. ${ }^{3}$ More recently, B. Cerjan et al. ${ }^{4}$ demonstrated the application of $\mathrm{Al}$ asymmetric nanoantenna in surface enhanced IR absorption (SEIRA) taking advantage of the alumina oxide layer to graft molecules at the nanoantenna surface. It is also known that the imaginary part of the $\mathrm{Al}$ dielectric constant exhibits a large absorption close to $800 \mathrm{~nm}$ that could induce a decrease of the plasmonics performances due to large damping of the plasmon resonance. However, in the studies performed by C. Langhammer et al. ${ }^{1}$ sharp plasmonic resonances were obtained in the near infra-red (NIR) indicating a potential for near-field enhancement in this spectral region. In this framework, we present a far-field and a near-field study of periodic aluminium nanocylinders arrays with different diameters. Those samples featured localised surface plasmon resonances that could be tuned from UV to NIR. We also observe multiple orders of resonance as well as strong Wood's anomalies in the plasmon excitation. The Wood anomalies are related to the Fano resonance coming from the interference of the plasmon mode of the nanocylinders and the grating modes of the array of nanocylinder. ${ }^{5}$ SERS was equally performed to quantify the near-field enhancement of those structures. Despite the high quality factor plasmonic resonators, we could not achieve a very high SERS activity. Advanced DDA calculations demonstrated that such structures could provide only limited near-field enhancement not sufficient for most of the SERS applications and not comparable to gold nanostructures even if their far-field optical properties are similar. 


\section{Experimental and Theoretical Methods}

\section{Experimental}

Square array of aluminium nanocylinders were fabricated by electron beam lithography in clean room. On top of a commercial $0.7 \mathrm{~mm}$ thick glass slide already covered with a $160 \mathrm{~nm}$ thick indium tin oxide (ITO) layer, a $140 \mathrm{~nm}$ thick Poly(methyl methacrylate) (PMMA) layer was deposited by spin coating. The PMMA layer was exposed with an electron beam using a ZEISS scanning electron microscope. The PMMA development was then done for $40 \mathrm{~s}$ in a standard 1:3 methyl isobutyl ketone / isopropanol (MIBK/IPA) solution. An aluminium layer of $30 \mathrm{~nm}$ was then deposited on the substrate. Finally a lift-off process was performed for one night in acetone to remove the PMMA.

The nanocylinder height was fixed to $30 \mathrm{~nm}$ and the nanocylinder diameters were varied from 50 to $250 \mathrm{~nm}$ by a $10 \mathrm{~nm}$ step. A gap of $200 \mathrm{~nm}$ was kept constant between the nanocylinders to avoid any near-field coupling. The quality of our nanostructures was verified by scanning electron microscopy in order to determine their exact sizes (figure 1).
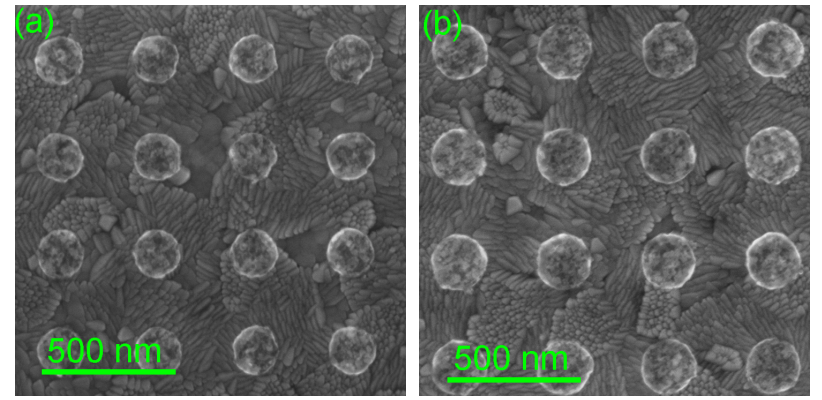

Figure 1: SEM images of arrays of aluminium nanocylinders produced by electron beam lithography for diameter of (a) $180 \mathrm{~nm}$ and (b) $205 \mathrm{~nm}$. The disks are deposited on a ITO coated glass substrate.

Extinction and SERS measurements were performed with an XploRA confocal Raman micro spectrometer (Horiba Scientific). For extinction measurements a non polarised white lamp was used to excite the nanocylinders and the transmitted light was collected with a $10 \times$ magnification objective (numerical aperture of 0.25 ) after removing the edge filters inside the 
Raman spectrometer. For SERS, the Raman spectra were recorded with $660 \mathrm{~nm}$ and $785 \mathrm{~nm}$ excitations wavelengths in backscattering configuration using a $100 \times$ magnification objective (numerical aperture of 0.90 ). The laser power was adjusted to $300 \mu \mathrm{W}$ with neutral density filter.

To measure the SERS signal, several probe molecules were chosen because of their high Raman cross-section. These molecules are thiophenol (TP), 1,2-di(4-pyridyl)ethylene (BPE) and methylene blue (MB). After UV-Ozone treatment the probe molecules were adsorbed on the nanocylinder samples sequentially. We started with the TP and finished with the MB. For all these probe molecules, the samples were immersed in a $1 \mathrm{mM}$ concentrated solution in ethanol for 1 hour and then dried. For each probe molecule, five Raman spectra were measured at different locations randomly distributed on each nanocylinder array. An average intensity was calculated by using the five Raman spectra and the standard deviation was used as error bar.

\section{Modeling}

The electric field calculation was performed by DDA (Discrete Dipole Approximation) ${ }^{6}$ using DDSCAT 7.3. ${ }^{7,8}$ The shape of the nanoparticles was generated by a home-made code written in Python. It consisted in arrays of nanocylinders of diameters going from $100 \mathrm{~nm}$ to $260 \mathrm{~nm}$ by a $20 \mathrm{~nm}$ step. Their upper corner had been rounded with a radius of curvature of $6 \mathrm{~nm}$ to take into account the imperfection of the lift-off process. The nanocylinders were arranged in a square lattice. The inter-particle spacing was set to $200 \mathrm{~nm}$.

The nanoparticle was discretized by dipoles separated by a distance of $1 \mathrm{~nm}$. The polarizability of each dipole was calculated by the filtered dipole as suggested by Piller and Martin. ${ }^{9}$ The inversion was carried out by biconjugate gradient with stabilization algorithm as proposed by Sleijpen and van der Vorst. ${ }^{10}$ Calculation was performed in double precision on the high performance calculation facilities PCIM (Pole Calcul Intensif pour la Mer). The convergence tolerance was set to $10^{-7}$. The nanoparticle was considered in an effective medium 
of relative permittivity as the mean of that of air and of ITO (Indium Tin Oxide). The former was considered constant and equal to 1 while the latter was measured by ellipsometry. The relative permittivity came from the paper of Rakić. ${ }^{11}$

The effect of a thin layer of alumina oxide $\left(\mathrm{Al}_{2} \mathrm{O}_{3}\right)$ around the nanocylinders was investigated following the approach proposed by Knight et al.. ${ }^{12}$ As the oxide layer appears the nanocylinders was considered as a mixture of oxide and aluminium whose relative permittivity was calculated with the Bruggeman model. The oxide refractive index was taken from ${ }^{13}$ (see supplementary information). The near-field features of the nanocylinders were calculated by averaging the electric-field in a shell of $1 \mathrm{~nm}$ thickness around the nanocylinders. This quantity is noted $\left\langle E^{2}\right\rangle$ hereafter.

\section{Results and discussion}

\section{Extinction analysis}
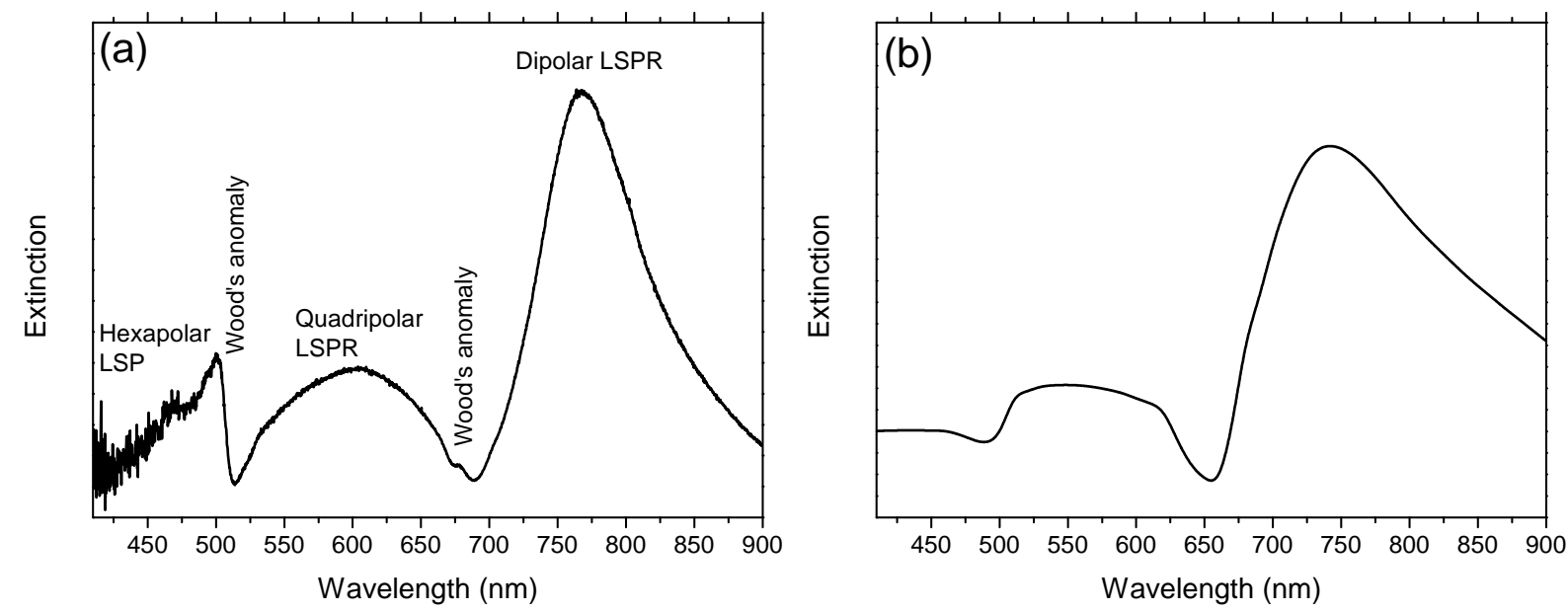

Figure 2: (a) Experimental and (b) simulated extinction spectra for nanocylinders array with diameter of $250 \mathrm{~nm}$.

The day after the nanocylinder production, the extinction spectrum of each nanocylinder diameter were recorded. The experimental extinction spectrum of $250 \mathrm{~nm}$ diameter nanocylenders is shown on figure 2a. It exhibits an intense dipolar mode at $770 \mathrm{~nm}$ as well as bands 
at lower wavelengths that could be assigned to higher orders of resonance (quadripolar and hexapolar modes). These latter bands are observable only for large diameters (higher than $80 \mathrm{~nm}$ for quadripolar mode and $210 \mathrm{~nm}$ for hexapolar one). LSPR position is plotted as a function of the nanocylinders diameter on figure 3. As expected, whatever the order of resonance is, the LSPR position red-shifts when the diameter monotonically increases as already observed for gold nanocylinders. ${ }^{14}$

The simulated extinction spectrum for nanocylinder diameter of $250 \mathrm{~nm}$ is shown in figure $2 \mathrm{~b}$. One can notice a very good agreement with the experimental spectrum and the simulation reproduces the different LSP modes. Such agreement is observable for all diameters as shown by the comparison of the simulated and experimental LSP positions on figure 3 .

The dipolar mode exhibits an asymmetric band shape because of the inter-band transitions of aluminium close to $800 \mathrm{~nm}$ (maximum of the imaginary part of the Al dielectric constant, figure 4). In the spectral range of the inter-band transitions, the damping of the plasmon increases and the extinction band broadens. The LSPR asymmetry is amplified by the Wood's anomaly observable as a drop of the extinction intensity on the low wavelength side of the LSPR. This anomaly is due to far field coupling between the individual nanocylinders through the array. ${ }^{15}$ The Wood's anomaly is clearly observable with those structures because of the ITO coating. Indeed, this latter one could induce the apparition of a guided mode at the substrate surface that amplifies the coupling between the nanostructures and as a consequence the Wood's anomaly observation. Very similar phenomenon was described by A. Christ et al. ${ }^{16}$

The LSPR broadening can be observed on figure 3 as the full width at half maximum of the dipolar mode is plotted as a function of its position. One can notice that the dipolar mode can be very thin (width lower than $50 \mathrm{~nm}$ ) and that the width increases strongly when the resonance becomes closer to the inter-band transitions at $800 \mathrm{~nm}$. This result is consistent with the intrinsic property of the aluminum. ${ }^{17}$ However, the widths are still comparable or even thinner than the ones measured for gold nanocylinders. ${ }^{18-20}$ This is actually remarkable 

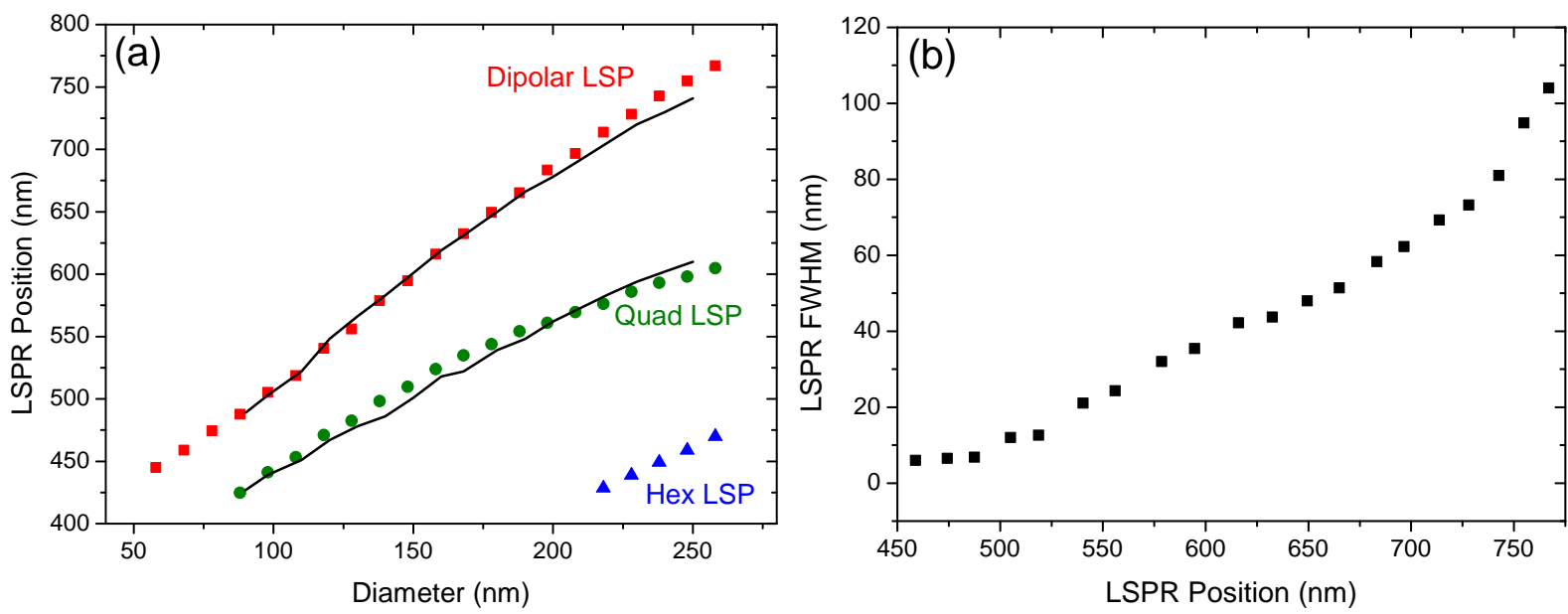

Figure 3: (a) LSPs position as a function of nanocylinders diameters. Dots correspond to the experimental measurements (red squares: dipolar mode, green dots: quadripolar mode, blue triangle: hexapolar mode) whereas the black lines correspond to DDA simulations. (b) Full width at half maximum of the dipolar mode as a function of the dipolar mode position.

since the imaginary part of $\mathrm{Al}$ is largely higher than the one of $\mathrm{Au}$ (between 10 and 50 times higher on the 600-900 nm range). This property could be exploited in sensors based on the observation of the LSPR shift due to the adsorption of molecules at the nanostructure surface and could confer to such systems a very high sensitivity

Moreover, the bandwidth has a direct influence on the Q-factor of the resonance. As previously demonstrated, ${ }^{18,21}$ a thinner LSPR band and as a consequence a larger the Q-factor of the plasmon resonator induces a greater near-field enhancement.

In the quasi-static approximation, Wang and $\operatorname{Shen}^{22}$ demonstrated that the quality $(Q)$ factor of the LSPR is given by:

$$
Q=\omega \frac{\frac{\partial \epsilon_{r}}{\partial \omega}(\omega)}{2\left(\epsilon_{i}(\omega)\right)^{2}}
$$

where $\epsilon_{r}$ and $\epsilon_{i}$ are the real and the imaginary parts of the dielectric constant of the material and $\omega$ is the pulsation. The application of this formula shows that in the visible range, the $\mathrm{Q}$ factor of aluminum nanoparticles is very low (lower than 0.5 for wavelength higher than $500 \mathrm{~nm}$ and nearly null around $800 \mathrm{~nm}$, fig.4a) ${ }^{17}$ whereas for gold, the Q factor is above 5 in the visible range and up to 25 for wavelength around $700 \mathrm{~nm}$. This is due to 
the largely higher imaginary part of the $\mathrm{Al}$ compared to $\mathrm{Au}$ in the visible range.

However, the Q-factor calculated experimentally from the extinction spectrum of $\mathrm{Al}$ nanocylinders can take values up to 70 for wavelengths lower than $500 \mathrm{~nm}$ (figure 5), which is of the same order of magnitude than for gold nanoparticles. The Q-factor decreases with the wavelength as the LSPR becomes closer to the inter-band transitions but is still higher than 5. This actually differs very much from the quasi-static approximation calculation probably thanks to the far-field coupling of LSPR with the grating, sharpening the extinction band and also to the fact that quasi-static approximation cannot be actually applied for big structures. In fact, the sharpening of the plasmon bands can be explained by two different effects: the far-field coupling of the nanocylinders ${ }^{5,23-25}$ and the Wood's anomalies. In the former case, the coupling leads to a reduction of the Full-Width at Half Maximum of the extinction band. In the latter cae, the Wood's anomaly is always located slightly blue shifted compared to the LSPR, inducing a possible coupling between those two modes and a supplementary sharpening of the LSPR. Thus, although the poor optical properties of alumina in the red, far-field properties of nanocylinders leads us to expect strong local field enhancement and then SERS detection capabilities even in the NIR region, when plasmon resonance is close to the inter-band transitions.
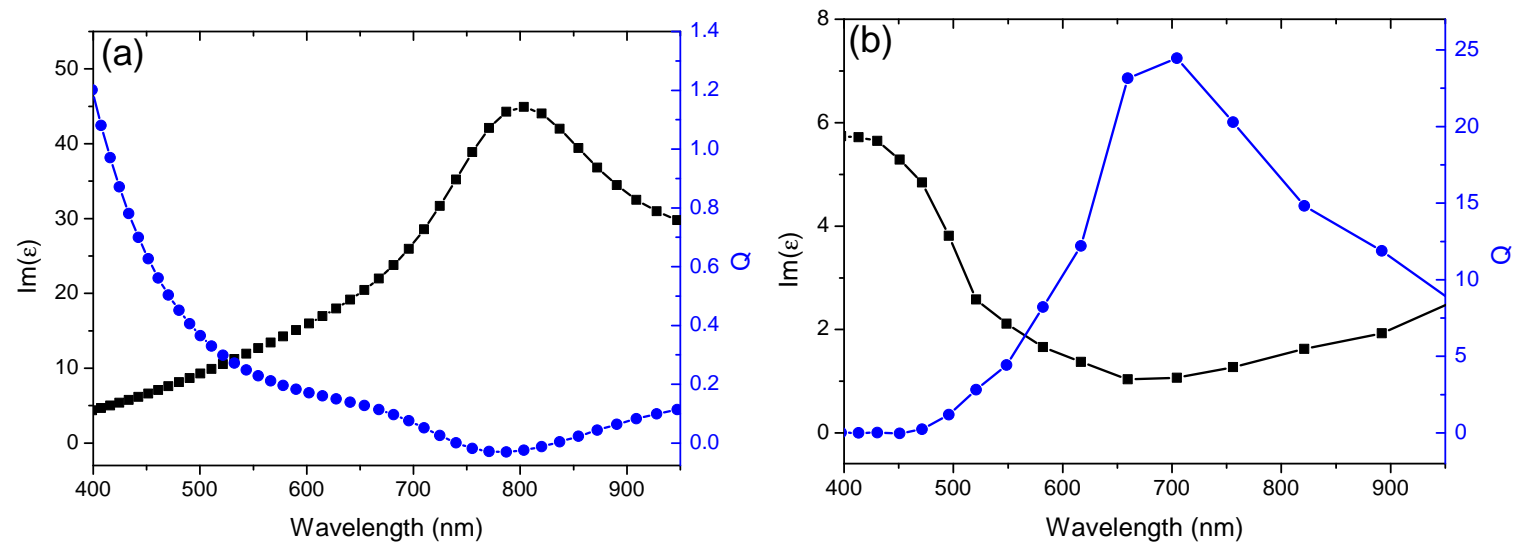

Figure 4: Imaginary part of the dielectric constant of Aluminium (black squares, left axis) and Q-factor in the quasi-static approximation (blue circles, right axis) with refractive index from Rakić 1998. (b) Same for gold with refractive index from Johnson and Christy. 


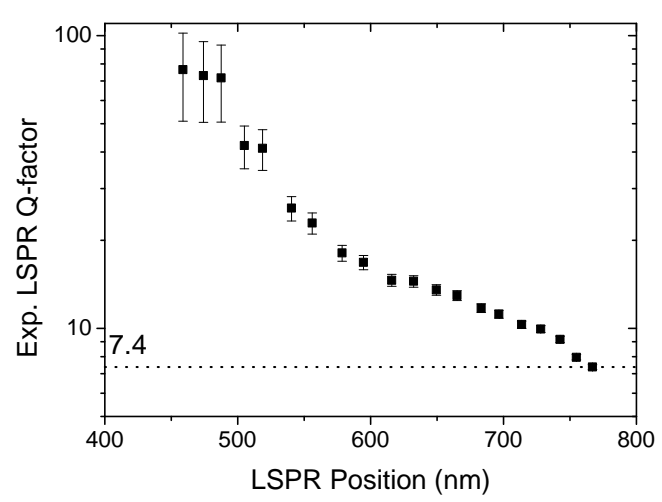

Figure 5: Experimental Q-factor calculated from the extinction spectra of the Al nanocylinders as a function of the LSPR position.

\section{SERS Analysis}

Several probe molecules were deposited sequentially at the surface of the nanocylinders (1st TP, 2nd BPE and 3rd MB) and SERS spectra were acquired at $660 \mathrm{~nm}$ and $785 \mathrm{~nm}$ excitation wavelengths for each nanocylinders diameter. For all diameters, none of the specific Raman bands of TP, BPE or MB were observed on the SERS spectrum as shown on figure 6a. This can be due to two distinct reasons. First, one can assume that the molecules cannot graft or adsorb at the nanocylinder surface and as a consequence, are not present at the surface of the nanocylinders during the SERS experiments. Second, the aluminium oxide layer prevented the molecules from attaining the near-field enhanced region. The oxide plays the role of a spacer layer between the molecules and the metallic surface. In both cases, we could not observe any SERS spectrum from the probe molecules that limit considerably the use of such metal for SERS.

However some Raman features in the low frequency range between 300 and $700 \mathrm{~cm}^{-1}$ can be observed, corresponding to the ITO substrate (figure 6). The intensity of these bands is enhanced in the presence of the nanocylinders and we used them to quantify the SERS enhancement. In figures 6(b) and 6(c), is shown the integrated SERS intensity from 300 to $700 \mathrm{~cm}^{-1}$ for $660 \mathrm{~nm}$ and $785 \mathrm{~nm}$ excitation wavelengths as a function of the LSPR positions. We observe a strong variation of the SERS signal when we tune the LSPR with a maximum 

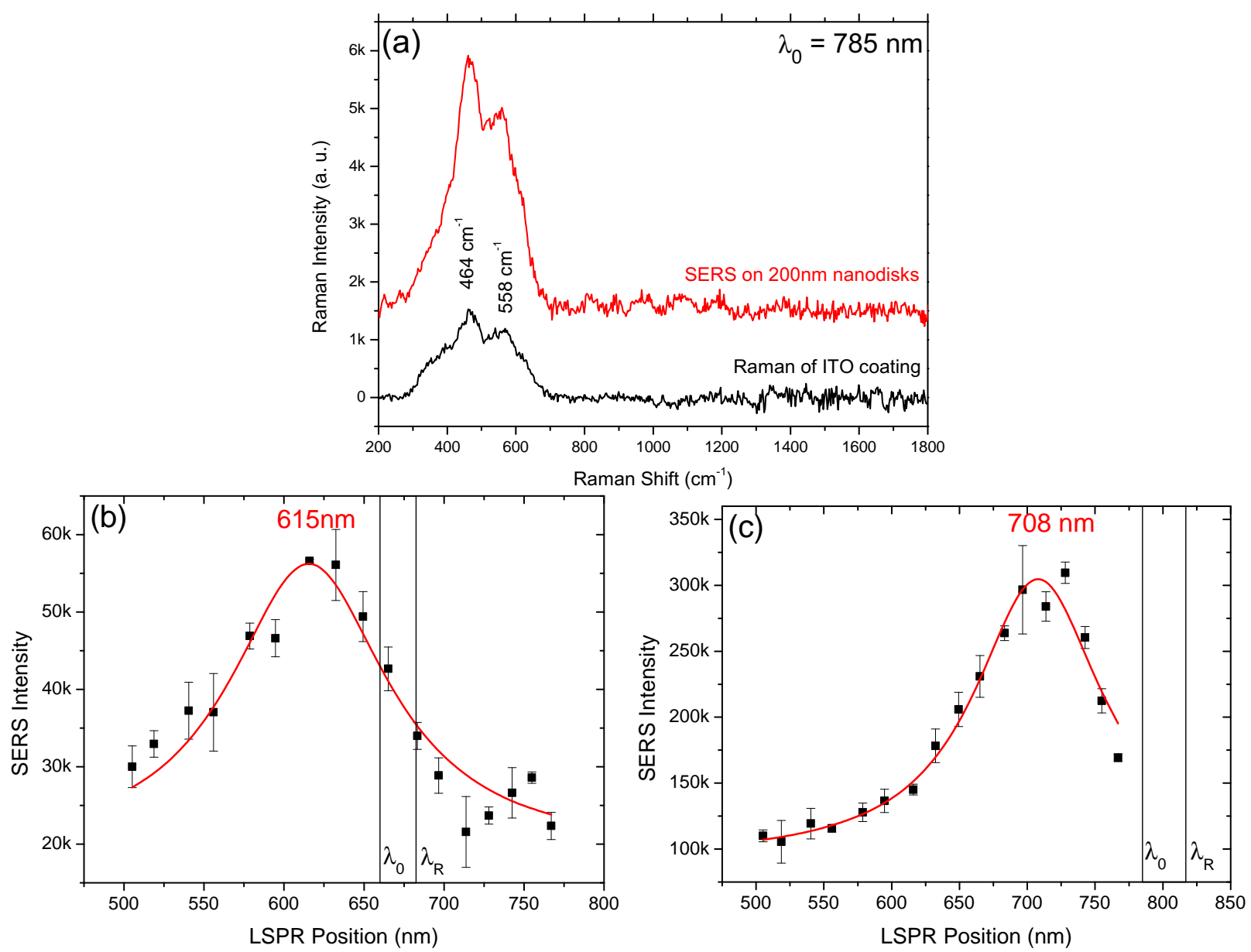

Figure 6: (a) Raman spectra on ITO coating and on nanocylinders array with the $785 \mathrm{~nm}$ excitation wavelength and SERS intensity as a function of the LSPR position for (b) $660 \mathrm{~nm}$ and (c) $785 \mathrm{~nm}$ excitation wavelengths. The black vertical lines correspond to the position of the excitation and Raman wavelengths $\left(\lambda_{0}\right.$ and $\lambda_{R}$ respectively).

of intensity at a specific LSPR. However, the SERS efficiency is lower than the one observed for gold since the ratio between the highest and the lowest signals is between 2 and 3 for $\mathrm{Al}$ whereas it is more than 10 for $\mathrm{Au} .{ }^{18}$ In addition, when we compare the size of the laser spot and the area of the nanocylinders, an enhancement less than 15 can be calculated in the case of the $200 \mathrm{~nm}$ diameter nanocylenders excited at $785 \mathrm{~nm}$. It is definitely much lower than typical SERS enhancement achieved with gold nanocylenders. ${ }^{26}$

This lower near-field enhancement with Al cannot be explained by a bad quality factor in this spectral range as we demonstrate in the previous section that the $\mathrm{Q}$ factor is high for our Al nanocylinders. 

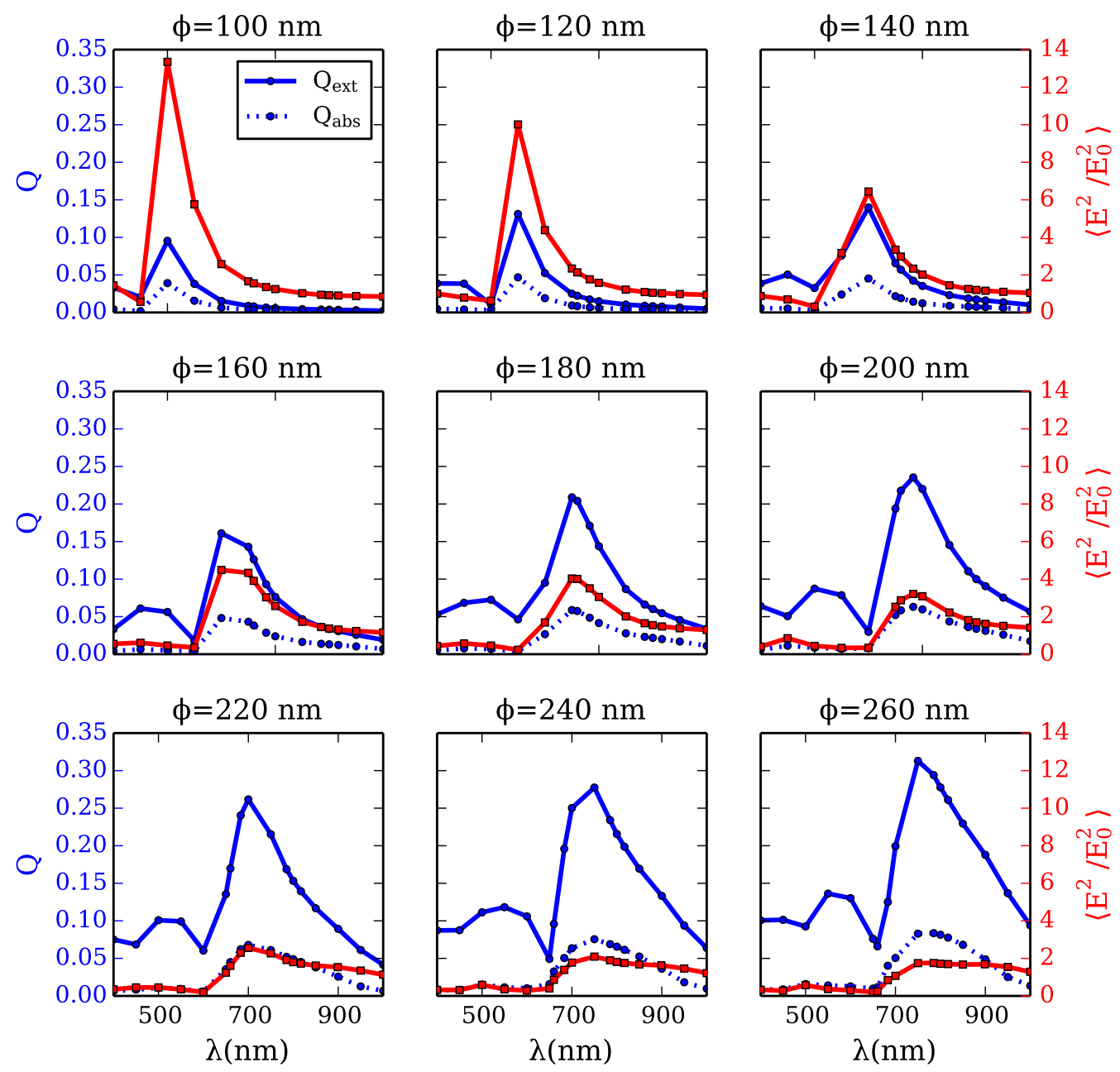

Figure 7: Near-Field feature of the Al nanocylenders: The near-field enhancement is plotted as $\left\langle E^{2} / E_{0}^{2}\right\rangle$ (red curve and right axis), the extinction cross section (blue curve and left axis) and the absorption cross section (blue dotted curve and left axis) are plotted versus the wavelength depending on the nanocylinder diameter $(\Phi)$.

This is confirmed by the DDA calculation. The average electric field around the nanocylenders is plotted on fig. 7 for diameters ranging from $100 \mathrm{~nm}$ to $260 \mathrm{~nm}$ and superimposed with the absorption and the extinction cross-section. First, the maxima of the absorption and the extinction cross-sections increase with the LSPR wavelength. Second, the maximum value of $\left\langle E^{2} / E_{0}^{2}\right\rangle$ decreases with the resonance wavelength, up to sevenfold when comparing the $100 \mathrm{~nm}$ and the $260 \mathrm{~nm}$ diameter nanocylenders. Third, for resonance wavelength larger than 
$700 \mathrm{~nm}$, the near-field spectrum appears wide and flat. These three main features can be explained by the inter-band transition. Indeed, the imaginary part of aluminium dielectric constant increases monotonically in the 400-700 nm range (figure 4). It has two consequences: first, an increase of the absorption and subsequently of the extinction cross-section, second, a decrease of the local field. In addition, the absorption maximum of the alumina is around $800 \mathrm{~nm}$ and at this wavelength, the plasmon mode is strongly absorbed.

The comparison of fig. 7 with previous work by Colas et al. ${ }^{18}$ shows that in the $600-800 \mathrm{~nm}$ domain $\left\langle E^{2} / E_{0}^{2}\right\rangle$ is an order of magnitude lower than in the case of gold nanocylenders, ${ }^{18}$ which lead to a lower SERS enhancement with aluminum as experimentally observed.

Moreover, the optimum LSPR position is largely outside the spectral range $\lambda_{0}, \lambda_{R}$ with $\lambda_{0}$ the excitation wavelength and $\lambda_{R}$ the Raman wavelength as the optimum LSPR positions to maximise the SERS signal is located at $615 \mathrm{~nm}$ for a $660 \mathrm{~nm}$ excitation wavelength and at $708 \mathrm{~nm}$ for a $785 \mathrm{~nm}$ excitation (figure 6). Previous works demonstrated experimentally and theoretically that the near-field can be red-shifted ${ }^{27-33}$ compared to the far-field resonance. This shift can reach up to $200 \mathrm{~nm}$ in the case of nanoantenna resonating in the mid-IR. ${ }^{29}$ However, figure 7 shows that the near-field is slightly shifted with regards to the far-field. This is similar to a previous study considering gold and aluminium nanocylinders ${ }^{18,34}$ in this spectral range. Indeed, the red-shift increases with the damping of the electron cloud oscillation inside the nanocylinder. ${ }^{31}$ It can be characterized by the Full Width at Half Maximum of the extinction bands. In the case of the nanocylinders, the FWHM increases from $20 \mathrm{~nm}$ to $100 \mathrm{~nm}$ as the diameter goes from 100 to $240 \mathrm{~nm}$. The losses associated with the nanocylinders are then expected to be small ${ }^{18}$ and the near-field to far-field red-shift subsequently low. Thus the assumption of a near-field to far-field shift cannot be considered to explain our SERS results.

As we previously noted, the absolute value of the $\left\langle E^{2} / E_{0}^{2}\right\rangle$ decreases and its band width increases when the LSPR is red-shifted. For example, for the $100 \mathrm{~nm}$ nanocylinder, the maximum value of $\left\langle E^{2} / E_{0}^{2}\right\rangle$ is around 14 with a bandwidth of $100 \mathrm{~nm}$ whereas the maximum 
value of $\left\langle E^{2} / E_{0}^{2}\right\rangle$ is only 3 for a $200 \mathrm{~nm}$ nanocylinder with a bandwidth around $130 \mathrm{~nm}$. Since the SERS intensity is proportional to the average Single Molecule Enhancement Factor ${ }^{17}$ $(\langle S M E F\rangle)$ defined by:

$$
\langle S M E F\rangle \propto \int_{V} \frac{E\left(\lambda_{0}\right)^{2} E\left(\lambda_{R}\right)^{2}}{E_{0}\left(\lambda_{0}\right)^{4}} \mathrm{~d} V
$$

where $E\left(\lambda_{0}\right)$ and $E\left(\lambda_{R}\right)$ are electric fields around the nanocylinders at the excitation and the Raman wavelengths respectively, $E_{0}$ is the incoming electric field and $V$ is the volume in which the calculation is carried out, the SERS intensity depends directly on the electric field around the nanocylinders. From fig. 7 we can assume that the SERS enhancement will be larger for the low resonance wavelengths even out of resonance compared to nanocylinders having higher resonance wavelength. For example, in the case of $\lambda_{0}=660 \mathrm{~nm},\left\langle E^{2}\left(\lambda_{0}\right) / E_{0}^{2}\right\rangle$ is larger for a diameter of $160 \mathrm{~nm}$ than a diameter of $180 \mathrm{~nm}$. However, the first resonates at $618 \mathrm{~nm}$ while the second at $656 \mathrm{~nm}$. This effect has already been observed and explained with gold nanocylenders. ${ }^{18}$

In addition, aluminium absorbs light in the $700-900 \mathrm{~nm}$ domain because of the inter-band transitions (Fig. 4). In this range, the electromagnetic energy is then converted to heat. ${ }^{34}$ This thermal effect is assumed to reduce electromagnetic enhancement traditionally express by eq. 2 .

These two effects contribute to reach a larger SERS signal with nanocylinder resonating at lower wavelengths and then to obtain an optimal LSPR position strongly blue shifted with regards to the excitation and the Raman wavelengths. The near-field enhancement is largely lower for Al than for $\mathrm{Au}$ as experimentally observed and this could also explain why we could not detect any SERS signal from the probe molecules, especially with the presence of the oxide layer at the nanocylinder surface. 


\section{Conclusion}

Aluminium nanocylinders were produced by electron beam lithography and their optical properties were investigated. We demonstrate that the LSPR can be tuned on the whole visible range. A broadening of the LSPR line width is observed when the LSPR is redshifted due to the inter band transitions occurring close to $800 \mathrm{~nm}$. We measured the near-field enhancement by SERS on probe molecules as well as on the ITO substrate. For the former ones no SERS signal was recorded due to very low enhancement and to the formation of the oxide layer. This means that such substrate could not be applied for SERS in this spectral range. The low enhancement is confirmed by the observation of the ITO SERS signal. The signal variation with the LSPR position is one order of magnitude lower than the one observed with gold. This enhancement is largely decreased when the LSPR is close to the inter band transition at $800 \mathrm{~nm}$ that results in a spectral shift between the SERS signal optimum and the LSPR position.

\section{Supporting Information}

Aeging study over two months of the aluminium nanocylinders arrays, using LSPR position.

\section{Acknowledgement}

The authors thank Tina Odaka and Denis Croizé-Fillon for their kind and efficient advices and help with the high calculation facilities. Thanks to the Centrale de Proximité en Nanotechnologies de Paris Nord (CPN2, 99 Avenue Jean-Baptiste Clément 93430 Villetaneuse, France) to supply the clean room for samples fabrication. The authors also acknowledge ANR P2N (ANR-12-NANO-0016) and the support of the French Government and ANRT Association Nationale de la Recherche et de la Technologie for funding. 


\section{References}

(1) Langhammer, C.; Schwind, M.; Kasemo, B.; Zoric, I. Localized Surface Plasmon Resonances in Aluminum Nanodisks. Nano Lett. 2008, 8, 1461-1471.

(2) Zhang, C.; Smirnov, A.; Hahn, D.; Grebel, H. Surface Enhanced Raman Scattering of Biospecies on Anodized Aluminum Oxide Films. Chem. Phys. Lett. 2007, 440, 239-243.

(3) Dörfer, T.; Schmitt, M.; Popp, J. Deep-UV Surface-Enhanced Raman Scattering. J. Raman Spectrosc. 2007, 38, 1379-1382.

(4) Cerjan, B.; Yang, X.; Nordlander, P.; Halas, N. J. Asymmetric Aluminum Antennas for Self-Calibrating Surface-Enhanced Infrared Absorption Spectroscopy. ACS Photonics 2016, 3, 354-360.

(5) Hicks, E. M.; Zou, S.; Schatz, G. C.; Spears, K. G.; Van Duyne, R. P.; Gunnarsson, L.; Rindzevicius, T.; Kasemo, B.; Käll, M. Controlling Plasmon Line Shapes Through Diffractive Coupling in Linear Arrays of Cylindrical Nanoparticles Fabricated by Electron Beam Lithography. Nano Lett. 2005, 5, 1065-1070.

(6) Purcell, E. M.; Pennypacker, C. R. Scattering and Absorption of Light by Nonspherical Dielectric Grains. Astrophys. J. 1973, 186, 705-714.

(7) Draine, B. T.; Flatau, P. J. Discrete-Dipole Approximation for Periodic Targets: Theory and Tests. $J$. Opt. Soc. Am. A 2008, 25, 2693-2703.

(8) Flatau, P. J.; Draine, B. T. Fast Near Field Calculations in the Discrete Dipole Approximation for Regular Rectilinear Grids. Opt. Express 2012, 20, 1247-1252.

(9) Piller, N.; Martin, O. J. Increasing the Performance of the Coupled-Dipole Approximation: A Spectral Approach. IEEE Trans. Antennas Propag. 1998, 46, 1126-1137.

(10) Sleijpen, G. L.; van der Vorst, H. A. Reliable Updated Residuals in Hybrid Bi-CG Methods. Computing 1996, 56, 141-163.

(11) Rakić, A. D. Algorithm for the Determination of Intrinsic Optical Constants of Metal Films: Application to Aluminum. Appl. Opt. 1995, 34, 4755-4767.

(12) Knight, M. W.; King, N. S.; Liu, L.; Everitt, H. O.; Nordlander, P.; Halas, N. J. Aluminum for Plasmonics. ACS Nano 2014, 8, 834-840, PMID: 24274662. 
(13) Palik, E. Handbook of Optical Constants of Solids II; Academic Press handbook series; Academic Press, 1991.

(14) Zorić, I.; Zäch, M.; Kasemo, B.; Langhammer, C. Gold, Platinum, and Aluminum Nanodisk Plasmons: Material Independence, Subradiance, and Damping Mechanisms. ACS Nano 2011, 5, 2535-2546, PMID: 21438568.

(15) Haynes, C. L.; McFarland, A. D.; Zhao, L.; Van Duyne, R. P.; Schatz, G. C.; Gunnarsson, L.; Prikulis, J.; Kasemo, B.; Käll, M. Nanoparticle Optics: The Importance of Radiative Dipole Coupling in TwoDimensional Nanoparticle Arrays. J. Phys. Chem. B 2003, 107, 7337-7342.

(16) Christ, A.; Tikhodeev, S.; Gippius, N.; Kuhl, J.; Giessen, H. Waveguide-Plasmon Polaritons: Strong Coupling of Photonic and Electronic Resonances in a Metallic Photonic Crystal Slab. Phys. Rev. Lett. 2003, 91, 183901.

(17) Le Ru, E. C.; Etchegoin, P. G. In Principles of Surface-Enhanced Raman Spectroscopy; Le Ru, E. C., Etchegoin, P. G., Eds.; Elsevier: Amsterdam, 2009; pp 121-183.

(18) Colas, F. J.; Cottat, M.; Gillibert, R.; Guillot, N.; Djaker, N.; Lidgi-Guigui, N.; Toury, T.; Barchiesi, D.; Toma, A.; Di Fabrizio, E. et al. Red-Shift Effects in Surface Enhanced Raman Spectroscopy: Spectral or Intensity Dependence of the Near-Field? J. Phys. Chem. C 2016, 120, 13675-13683.

(19) Lamy de la Chapelle, M.; Shen, H.; Guillot, N.; Frémaux, B.; Guelorget, B.; Toury, T. New Gold Nanoparticles Adhesion Process Opening the Way of Improved and Highly Sensitive Plasmonics Technologies. Plasmonics 2013, 8, 411-415.

(20) Shen, H.; Guillot, N.; Rouxel, J.; Lamy de la Chapelle, M.; Toury, T. Optimized Plasmonic Nanostructures for Improved Sensing Activities. Opt. Express 2012, 20, 21278-21290.

(21) Colas, F.; Barchiesi, D.; Kessentini, S.; Toury, T.; Lamy de la Chapelle, M. Comparison of Adhesion Layers of Gold on Silicate Glasses for SERS Detection. J. Opt. 2015, 17, 114010.

(22) Wang, F.; Shen, Y. R. General Properties of Local Plasmons in Metal Nanostructures. Phys. Rev. Lett. 2006, 97, 206806.

(23) Meier, M.; Wokaun, A.; Liao, P. F. Enhanced Fields on Rough Surfaces: Dipolar Interactions Among Particles of Sizes Exceeding the Rayleigh Limit. J. Opt. Soc. Am. B 1985, 2, 931-949. 
(24) Carron, K. T.; Fluhr, W.; Meier, M.; Wokaun, A.; Lehmann, H. W. Resonances of Two-Dimensional Particle Gratings in Surface-Enhanced Raman Scattering. J. Opt. Soc. Am. B 1986, 3, 430-440.

(25) Félidj, N.; Laurent, G.; Aubard, J.; Lévi, G.; Hohenau, A.; Krenn, J. R.; Aussenegg, F. R. GratingInduced Plasmon Mode in Gold Nanoparticle Arrays. J. Chem. Phys. 2005, 123, 221103.

(26) David, C.; Guillot, N.; Shen, H.; Toury, T.; Lamy de la Chapelle, M. SERS Detection of Biomolecules Using Lithographed Nanoparticles Towards a Reproducible SERS Biosensor. Nanotechnology 2010, 21, 475501.

(27) Ross, B. M.; Lee, L. P. Comparison of Near- and Far-Field Measures for Plasmon Resonance of Metallic Nanoparticles. Opt. Lett. 2009, 34, 896-898.

(28) Kelly, K. L.; Coronado, E.; Zhao, L. L.; Schatz, G. C. The Optical Properties of Metal Nanoparticles: The Influence of Size, Shape, and Dielectric Environment. J. Phys. Chem. B 2003, 107, 668-677.

(29) Alonso-González, P.; Albella, P.; Neubrech, F.; Huck, C.; Chen, J.; Golmar, F.; Casanova, F.; Hueso, L. E.; Pucci, A.; Aizpurua, J. et al. Experimental Verification of the Spectral Shift between Near- and Far-Field Peak Intensities of Plasmonic Infrared Nanoantennas. Phys. Rev. Lett. 2013, 110, 203902.

(30) Bryant, G. W.; de Abajo, F. J. G.; Aizpurua, J. Mapping the Plasmon Resonances of Metallic Nanoantennas. Nano Lett. 2008, 8, 631-636.

(31) Zuloaga, J.; Nordlander, P. On the Energy Shift between Near-Field and Far-Field Peak Intensities in Localized Plasmon Systems. Nano Lett. 2011, 11, 1280-1283.

(32) Kats, M. A.; Yu, N.; Genevet, P.; Gaburro, Z.; Capasso, F. Effect of Radiation Damping on the Spectral Response of Plasmonic Components. Opt. Express 2011, 19, 21748-21753.

(33) Moreno, F.; Albella, P.; Nieto-Vesperinas, M. Analysis of the Spectral Behavior of Localized Plasmon Resonances in the Near- and Far-Field Regimes. Langmuir 2013, 29, 6715-6721.

(34) Picardi, G.; Colas, F. J.; Gillibert, R.; Lamy de la Chapelle, M. Spectral Shift of the Plasmon Resonance Between the Optical Extinction and Absorption of Gold and Aluminum Nanodisks. J. Phys. Chem. C 2016, 

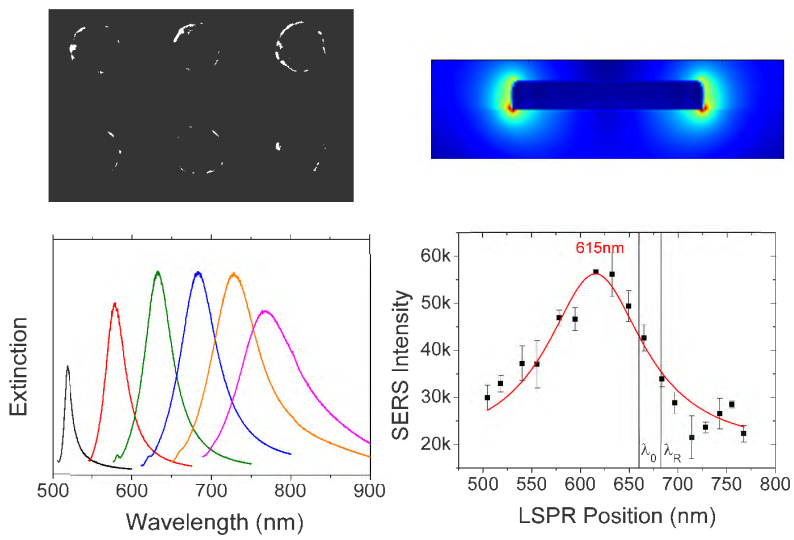

TOC graphic 